\title{
UV ENERGY CURING OF DIELECTRIC LAYER FOR SCREEN PRINTED CAPACITIVE CHEMICAL SENSORS
}

\author{
Miha Golob (D) \\ The Secondary School of Multimedia and Graphic Technology Ljubljana, Ljubljana, Slovenia
}

\begin{abstract}
Functional printing is becoming a new standard in the printing industry and new materials are being developed for use with conventional printing methods. The purpose of our research was to successfully print and measure the change in capacitance of a multi-layered interdigitated capacitor, when exposed to water vapour in air. Commercially available printing inks were applied, including one silver-based conductive ink and one dielectric ink. Conductive structures with resolution of up to 300 microns were printed with a screen density of 120 lines $/ \mathrm{cm}$. Two-layered elements of dielectric printing ink and an additional layer of conductive ink were successfully applied onto a printing substrate coated with a conductive indium tin oxide layer. Capacitance of a parallel-plate and interdigitated capacitor was determined by implementing variation in the position of electrodes for measurements. The results confirm that the change of UV energy applied for curing of the dielectric ink has no significant influence on the capacitance of printed sensors, as opposed by the factor of capacitor function and surface area. Capacitance was greater when measured as a parallel-plate capacitor with dielectric layer between two electrodes and a larger surface area than interdigitated in-plane capacitor printed on the same sample. Dissipation factor diminishes with higher UV energy applied for curing of the dielectric ink. Sensor response to changes in relative humidity is even and can be reproduced. Change of capacitance of sensor is higher with increase in relative humidity, thus the prepared sensors are properly responsive.
\end{abstract}

Key words: screen printing, chemical sensor, functional printing, UV energy

\section{INTRODUCTION}

Printed electronics is a technology that merges electronics manufacturing and text/graphic printing. By this combination it is feasible to manufacture high-quality electronics products that are thin, flexible, wearable, lightweight, of varying sizes, ultra-cost-effective, and environmentally friendly. Fabrication of various types of sensing devices is seen as one of many possible applications in the field of printed electronics, for example, environmental sensors (temperature, humidity, gas concentration, ion concentration), biosensors (glucose, blood pressure, DNA), pressure sensors (floor mat sensor, touch sensor, explosion sensor), and light sensors (Suganuma, 2014).

Among different sensing principles, sensors based on resistance changes-chemoresistors, and capacitive changes-chemocapacitors, have attracted considerable research interest because they fulfil the low energy consumption criterion. Both sensor types can be fabricated with conventional microelectronicmicromachning processes as well as functional printing and they operate at room temperature offering low power consumption. Their response is usually fast and reversible and present good sensitivity to polar analytes (Botisalas et al, 2013).

The operating principle of planar interdigitated capacitance sensor (IDCS) basically follows the rule of two parallel plate capacitors, where electrodes open up to provide a one sided access to material under test (MUT) [Abdul Rahman et al, 2014]. In an IDCS the sensitivity to variations of the conductivity or permittivity depends on the penetration of the electric field inside a MUT contributing to the capacitance and impedance between electrodes (Mamishev et al, 2004). Depending on the geometric configuration of the electrodes the electric field lines can penetrate deeper or less deep. The capacitance of an IDCS always depends on the dielectric properties of the MUT and the geometry of the electrodes (Guadarrama-Santana et al, 2014). When the sensitive layer (commonly a polymer) interacts with the chemicals present in the environment, the chemically sensitive layer changes its conductivity $(\sigma)$, dielectric constant $(\varepsilon)$ or its effective thickness $(d)$. The interdigitated capacitance (IDC) chemical sensor can then detect a change in capacitance and/or impedance due to a change of the dielectric constant and thickness of the layer. IDC chemical sensors have been investigated by many researchers because they are inexpensive to manufacture and easily integrated with other sensing components and signal processing electronics (Mamishev et al, 2004).

Since the electrodes of an IDCS are coplanar, the measured capacitance will give a high signal-to-noise ratio. Consequently, the electrode pattern of the IDCS can be repeated many times to get a strong signal. 
The term "interdigitated" refers to a digit-like or finger-like periodic pattern of parallel in-plane electrodes, used to build up the capacitance associated with the electric fields that penetrate into a material sample (Abdul Rahman et al, 2014).

Chemical sensors constitute a large portion of all interdigital sensors described in the literature. They are used in detection of various gases, chemicals, moisture, organic impurities, etc. A typical chemical interdigital sensor design is to deposit interdigital electrodes on an insulating substrate (Mamishev et al, 2004). Application of electrodes can be achieved by using common printing methods such as screen printing. Polymer inks containing electrically conductive particles are the most common choice for this purpose in the field of printed electronics, with silver particles offering the lowest resistivity (Žveglič, 2011). The electrodes are then coated with a thin layer of material that is sensitive to the concentration of chemicals present in the ambient atmosphere. When the sensor is exposed to ambient chemicals, changes in capacitance are the result of the change of dielectric constant and effective thickness of the sensitive layer (Mamishev et al, 2004). Coating material often consists of derivatives of metacrylic acid, such as Poly(2-hydroxyethyl metacrylate) or pHEMA (Reddy, 2011), Poly(butyl methacrylate) or PBMA and Poly(ethyl methacrylate) or PEMA (Oikonomou et al, 2011), Polyvinylpyrrolidone or PVP, Poly(ethyleneimine) or PEI, polyhydroxystyrene or PHS (Botisalas et al, 2013).

\section{METHODS}

Electrodag PM-470 conductive screen-printable ink (Acheson Colloiden B.V., Netherlands) was used. It contains finely distributed silver particles in a thermoplastic resin. Its density is about $2140 \mathrm{~kg} / \mathrm{m}^{3}$ and the solid content 58-62\%. CFSN6057 Suntronic Dielectric 681 dielectric screen-printable ink with density of approximately $1270 \mathrm{~kg} / \mathrm{m}^{3}$, dielectric constant specified at $\varepsilon=4.76$ (@ $1 \mathrm{MHz}$ ) and breakdown voltage using direct current at $3100 \mathrm{~V}$ at layer thickness $25,4 \mu \mathrm{m}$. Recommended curing of the dielectric ink by the manufacturer is $650 \mathrm{~mJ} / \mathrm{cm}^{2}$.

RokuPrint SD 05 semi-automatic screen printing machine with adjustable table KOO3 and pneumatic squeegee mechanism PR01 was used for printing. Printing forms with resolution of conductive structures of up to 300 microns were prepared on a high-modulus monofilament polyester plain weave mesh $120 / 34$ Y. A squeegee with a hardness of $75^{\circ}$ Sh was used. For printing substrate, clear polyethylene film, coated on one side with indium tin oxide (ITO) was used.

Double layer prints (wet-on-dry) of dielectric ink (CFSN6057 Suntronic Dielectric 681) were made on conductive side of ITO coated film. Two samples of four different double layer prints were prepared and each layer of the sample cured with UV energy of 600,1200, 1800, and $2400 \mathrm{~mJ} / \mathrm{cm}^{2}$, respectively. UV curing was carried out using Aktiprint L 10-1 UV dryer. Additional layer of conductive ink was printed (weton-dry) on top of the dielectric layer for each sample. The drying procedure involved placing the printed substrate level on a work bench for $5 \mathrm{~min}$ at room temperature and curing at $170{ }^{\circ} \mathrm{C}$ for $5 \mathrm{~min}$ in infrared tunnel Shrink Machine BS-B400.

The measurement of the change in capacitance of prepared sample sensors was carried out using Boonton 72B capacitance meter at the frequency of $1 \mathrm{MHz}$. Sample response to relative humidity was carried out in a measurement chamber with approximately $1 \mathrm{~L}$ volume after first neutralizing the measurement chamber with nitrogen gas with gas flow of $1,1 \mathrm{~L} / \mathrm{min}$. All measurements were conducted at temperature of $26^{\circ} \mathrm{C}$.

\section{RESULTS}

\subsection{Capacitance measurements}

Figure 1 depicts the placement of electrodes used in capacitance measurements of prepared sensor. The dielectric layer was placed between a conductive layer of indium tin oxide (ITO) on the surface of the substrate and a geometry of interdigitated sensor electrodes screen printed with conductive ink in the same plane (Lo, HiS, HiR, and Sb) on the surface of the dielectric layer.

Two different capacitor configurations were used, based on the position of the electrodes and dielectric layer. Measurements between electrodes Lo-ITO and Sb-ITO were carried out using parallel plate configuration. Measurements between electrodes Lo-HiS and Lo-HiR had the characteristics of an interdigitated capacitor (Figure 2). 
Top view

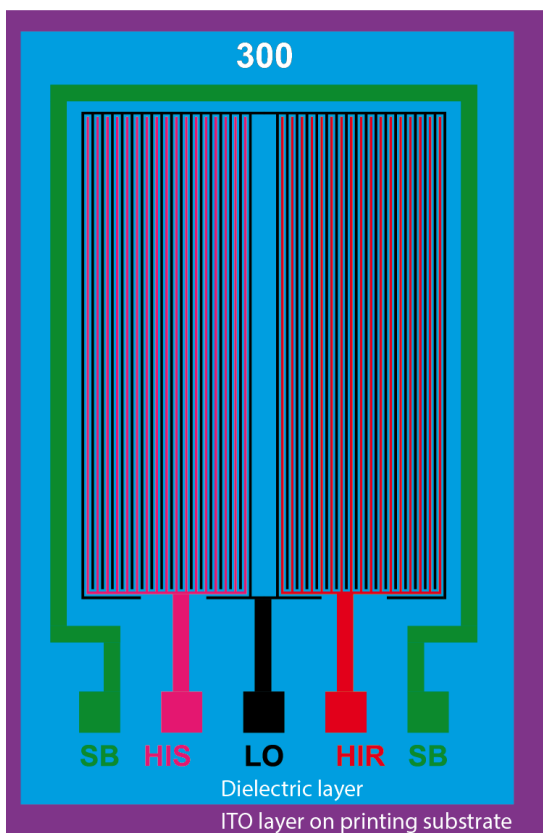

Side view
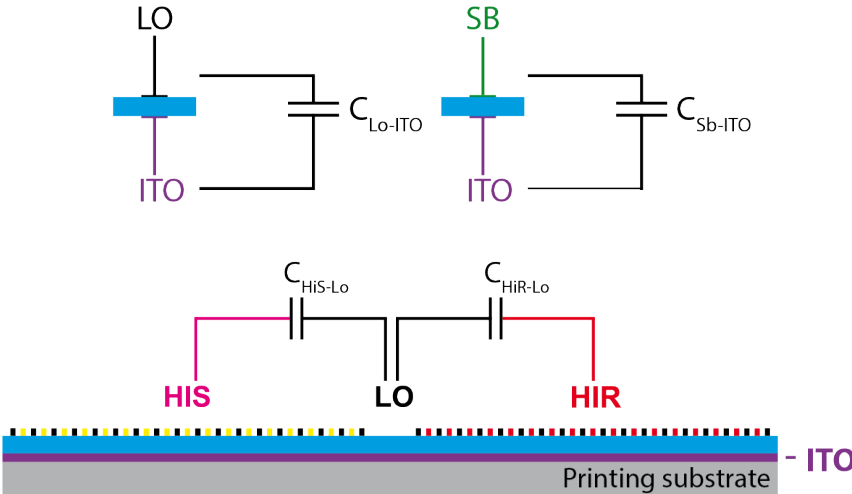

Legend

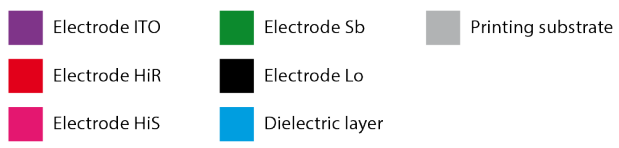

Figure 1: Schematic view of sensor electrode and dielectric layer placement.

Lo-HiS

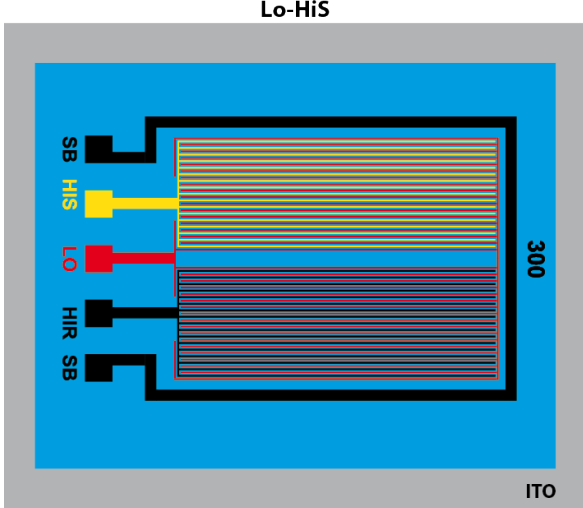

Lo-ITO

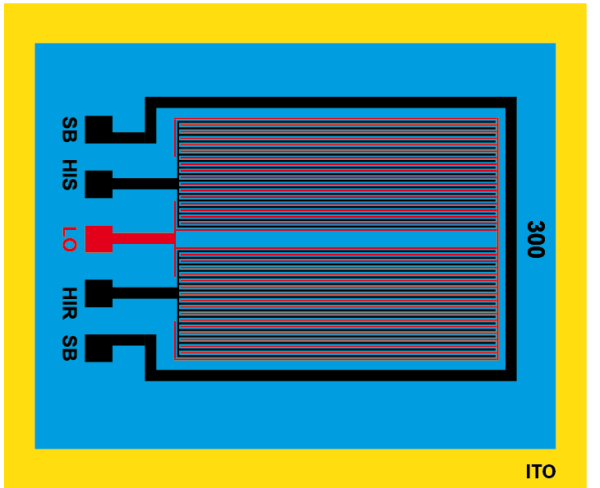

Lo-HiR

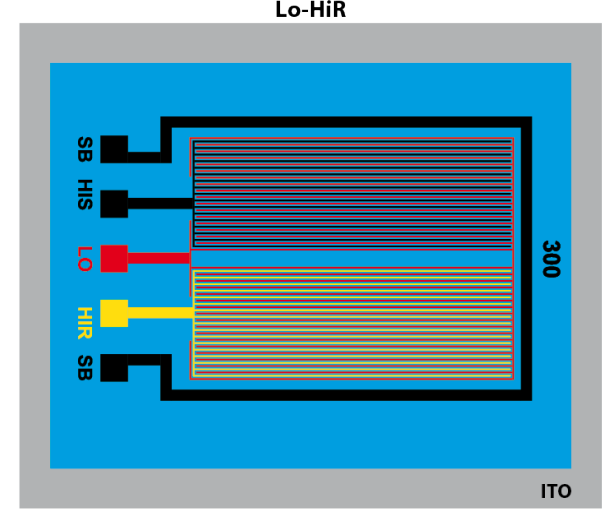

Sb-ITO

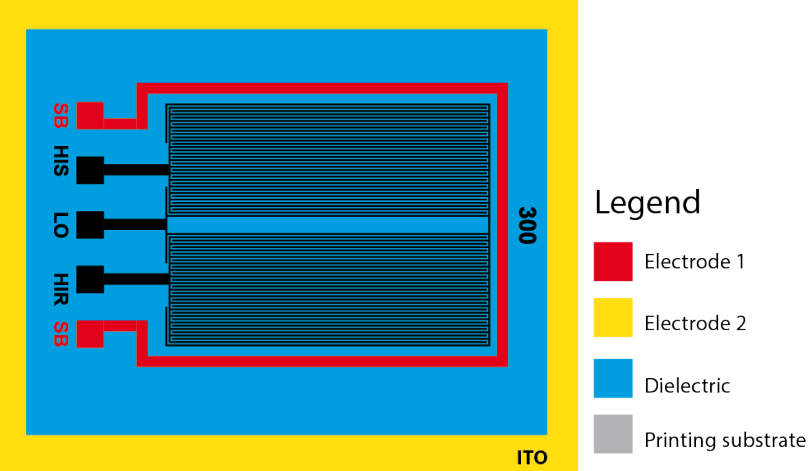

Figure 2: Electrode configuration for capacitance measurement.

Figure 3 shows the results of capacitance of prepared capacitors in relation to configuration of electrodes for measurement and UV energy used for curing of the dielectric layer. Out of eight prepared sensors, we experienced only one shortcircuit of a sample cured with UV energy of $600 \mathrm{~mJ} / \mathrm{cm}^{2}$. Measurements show that the influence of UV energy used for curing of the dielectric functional ink is small. On the other hand 
we observed an increase in capacitance with the increase of surface of the electrodes. For parallel-plate capacitors with a dielectric layer between electrodes, capacitance $C$ is defined as:

$C=\varepsilon \varepsilon_{0} \frac{A}{d},[\mathrm{pF}]$

where $A$ is the surface of the electrodes, $d$ is the thickness of dielectric layer, $\varepsilon$ is the dielectric constant, $\varepsilon_{0}$ is constant value defined as $\varepsilon_{0}=8,854 \cdot 10^{-12} \mathrm{~F} \mathrm{~m}^{-1}$ (Breurer et al, 1993; Horvat, 2015). The capacitance of parallel-plate Lo-ITO capacitor is approximately 50 \% greater than the capacitance of parallel-plate Sb-ITO configuration and approximately $70 \%$ greater than the average of Lo-HiS/Lo-HiR interdigitated capacitor measurements. The latter is shown as an average since both capacitors use identical geometry and differences in measurements were negligible. Higher capacitance of Lo-ITO configuration may be caused by some advantages of interdigitated sensor design on one side and increased surface on the other, when compared to measurement results of capacitance between electrodes Sb-ITO and Lo-HiS/HiR.
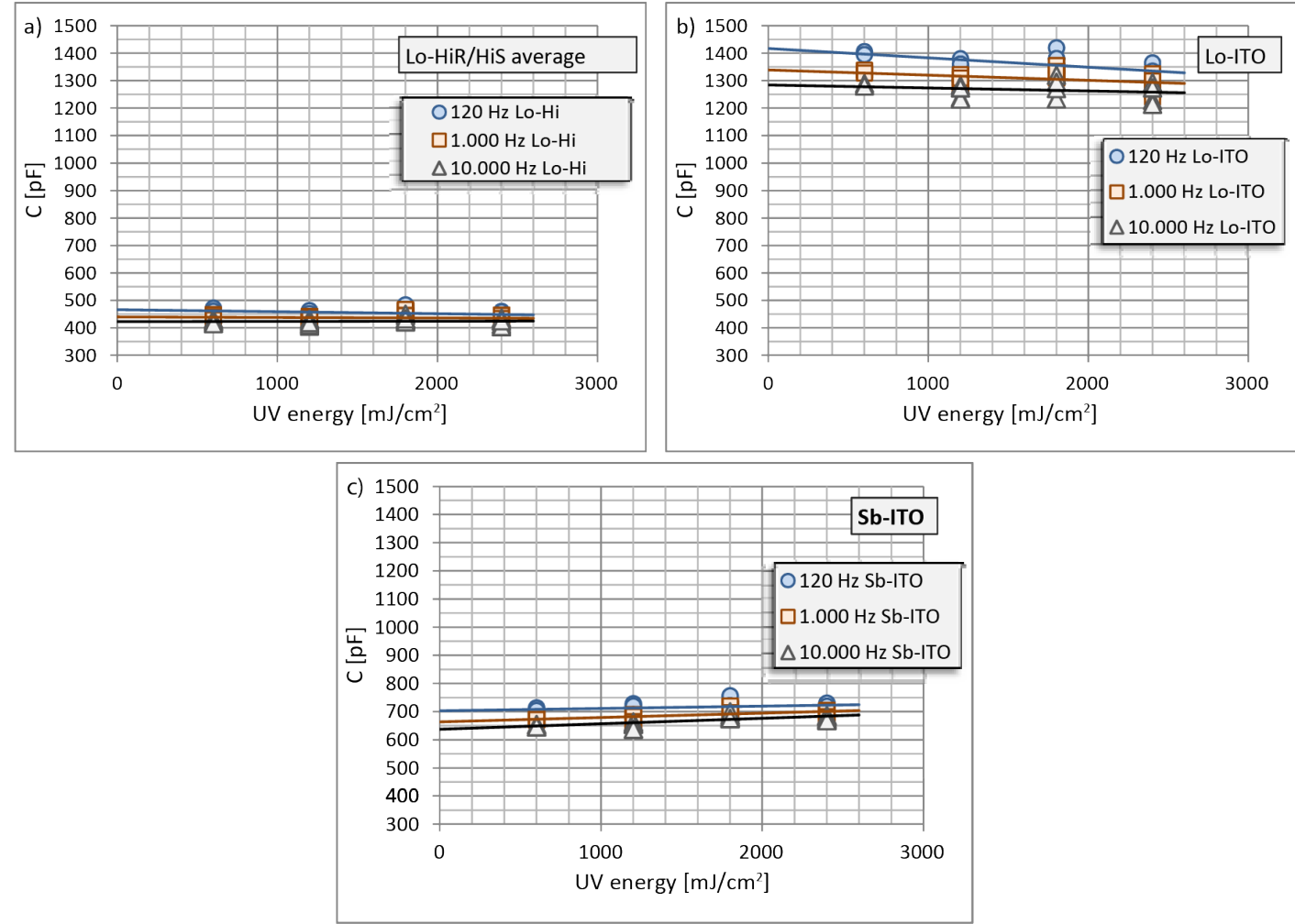

Figure 3: Capacitance measurement of screen-printed capacitors at different frequencies and different configuration of electrodes for measurement: (a) Lo-HiS/Lo-HiR average, (b) Lo-ITO, (c) Sb-ITO.

Surface area of different electrodes used in measurements is presented in figure 4.

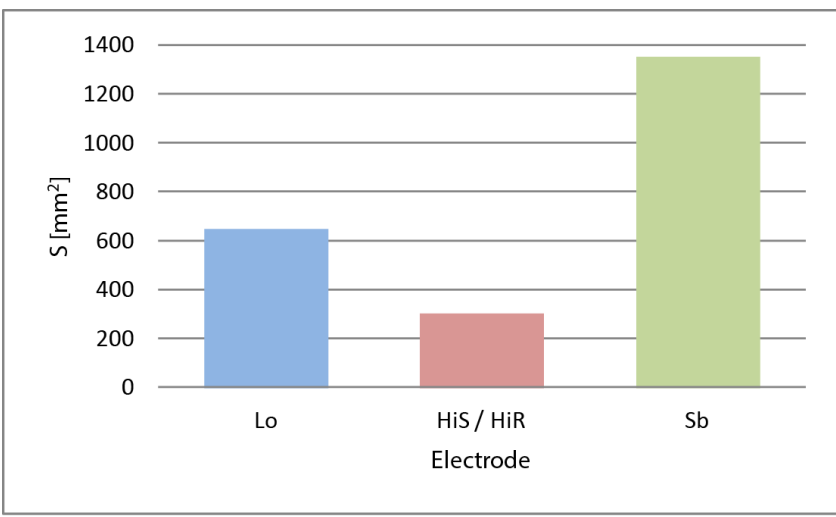

Figure 4: Surface of electrodes used in capacitance measurements (from left to right: Lo, HiS/HiR, Sb). 


\subsection{Dissipation factor}

Dielectric loss quantifies a dielectric material's inherent dissipation of electromagnetic energy (e.g. heat). In a capacitor made of a dielectric placed between conductors it is quantified as the dissipation factor (DF), a measure of loss-rate of energy of a mode of oscillation in a dissipative system. It is the reciprocal of quality factor, which represents the "quality" or durability of oscillation. It can be parameterized in terms of either the loss angle $\delta$ or the corresponding loss tangent tan $\delta$ (Horvat, 2015).

Based on the specification provided by dielectric ink manufacturer, the dissipation factor $(\tan \delta)$ corresponds to DF =0.013 (@1 MHz). Based on the measurements represented in figure 5 we can observe that the dissipation factor diminishes with increase in UV energy used for curing of dielectric layer and an increase in frequency of measurement. The biggest variations in measurements were observed when frequency of $120 \mathrm{~Hz}$ was used.
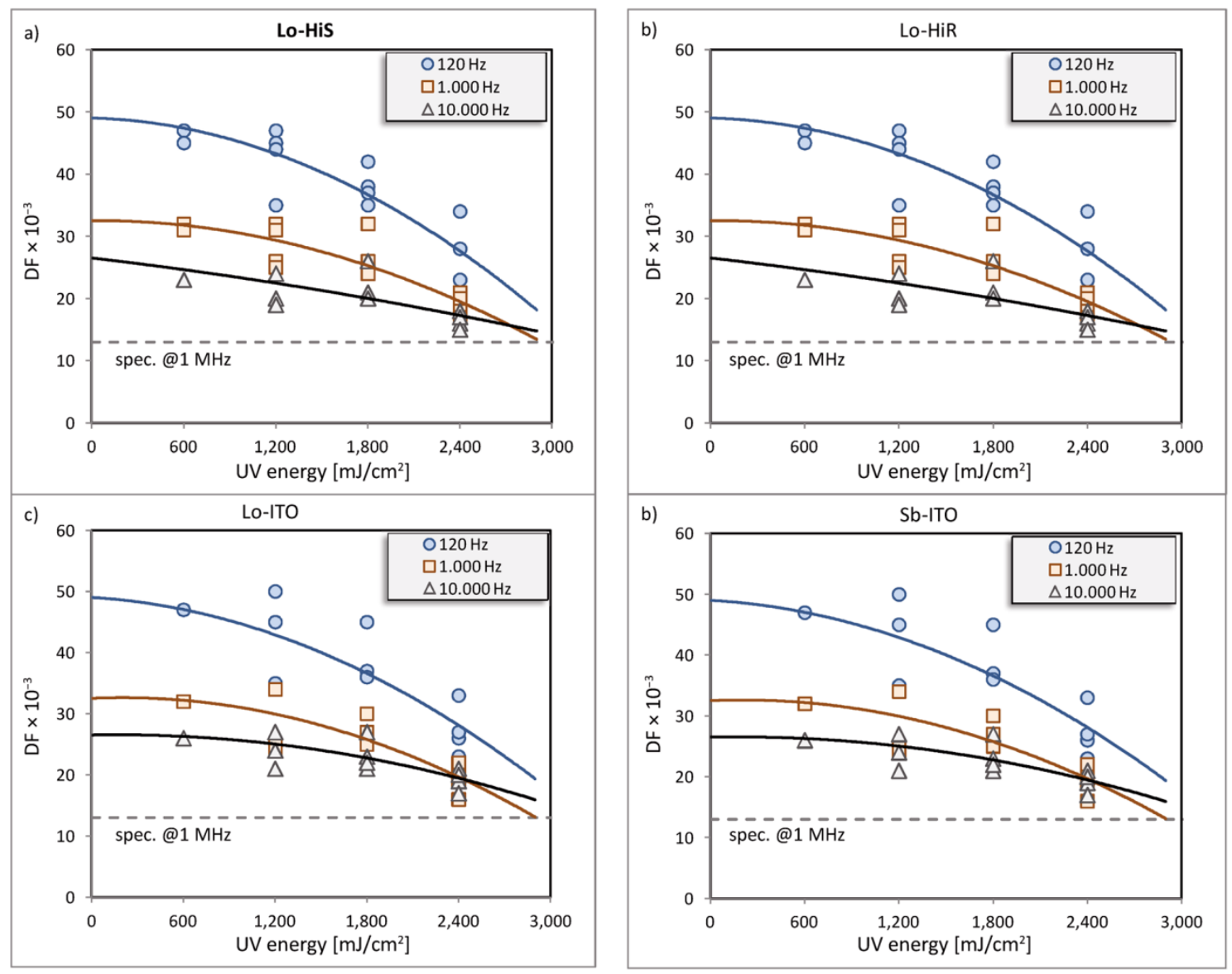

Figure 5: Dissipation factor measurements of screen-printed capacitors at different frequencies and different configuration of electrodes for measurement (a) Lo-HiS, (b) Lo-HiR, (c) Lo-ITO, (d) Sb-ITO.

\subsection{Sensor response to change in relative humidity}

Sensor with dielectric layer that was cured at UV energy of $2400 \mathrm{~mJ} / \mathrm{cm}^{2}$ was considered to be the most appropriate to study the response to changes in relative humidity, based on the measurements of capacitance and dissipation factor (Figure 6). In-plane interdigitated electrode geometry Lo-HiR, was used for measurements of sensor response.

First measurements of sensor response were made at relative humidity of $45 \%$. The base value of capacitance when the measurement chamber was filled with nitrogen gas was in the range of $250 \mathrm{pF}$. When water vapour was introduced to the measurement chamber the capacitance slowly increased to approximately 340 pF (figure 7). In comparison, the drop in capacitance was quicker, when the chamber was being purged with nitrogen gas. Sensor was characterized in transient state with six consecutive measurements made and all of them showed a similar response in change of capacitance. 


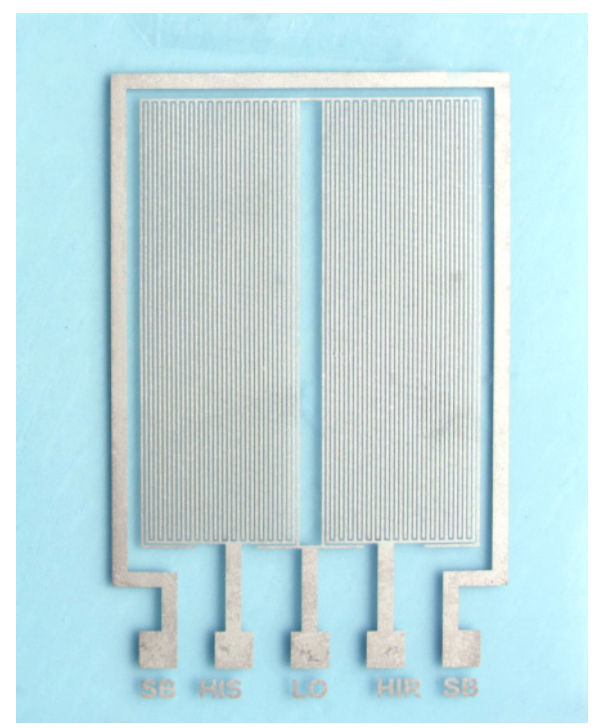

Figure 6: Sample sensor with conductive 300 micron interdigitated structure screen printed on two layer dielectric cured with UV energy $2400 \mathrm{~mJ} / \mathrm{cm}^{2}$.

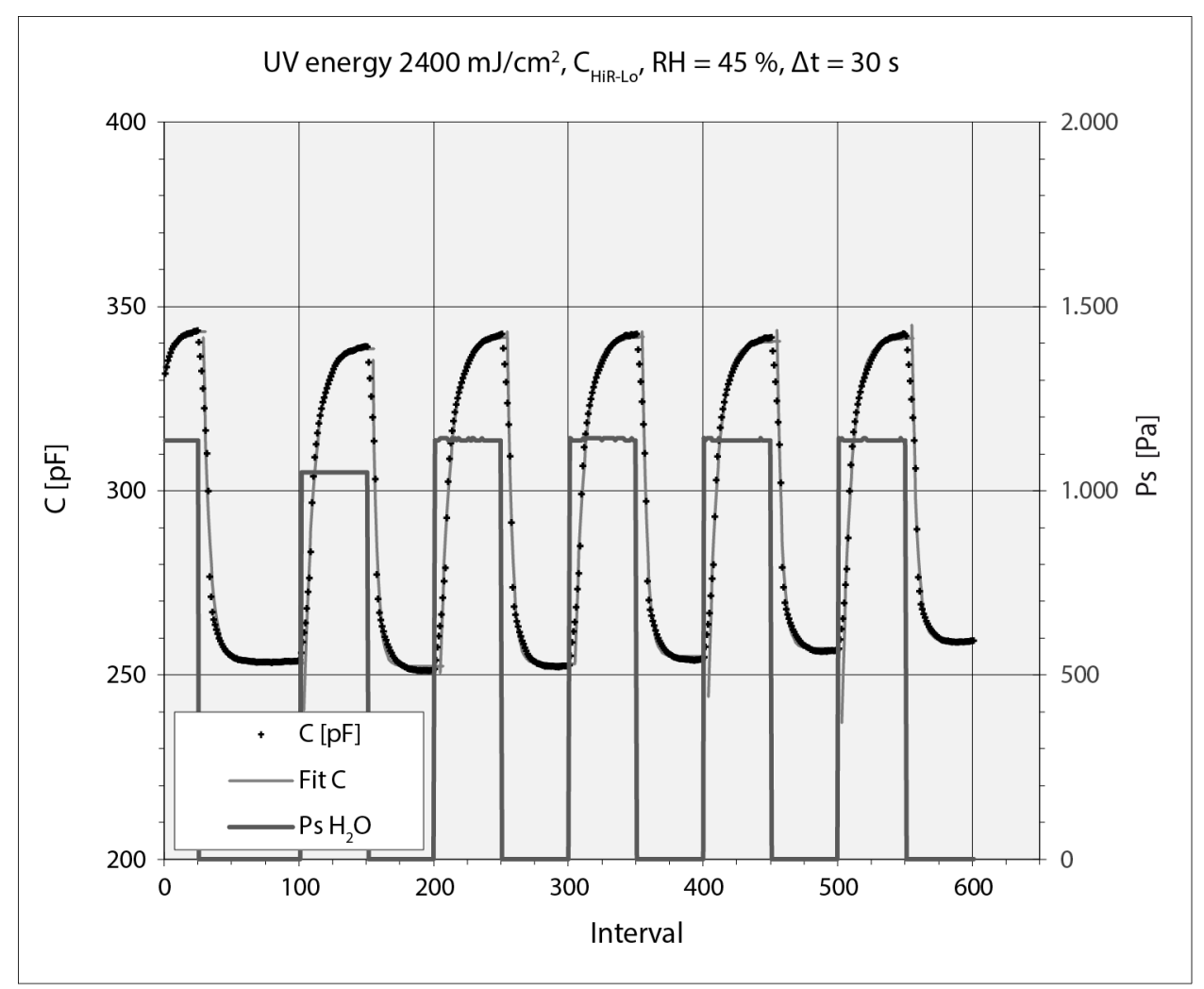

Figure 7: Sensor response to the change in relative humidity of $45 \%$ in the measurement chamber.

Second measurements of sensor response were made at relative humidity of $53 \%$. The base value of capacitance when the measurement chamber was filled with nitrogen gas was in the range of $260 \mathrm{pF}$. When water vapour was introduced to the measurement chamber the capacitance slowly increased to approximately $370 \mathrm{pF}$ (figure 8). This shows that sensor response was greater when higher values of relative humidity were introduced and that the sensor is properly responsive. Six consecutive measurements were made. First and second measurements of capacitance differ from the rest, due to an error on the nitrogen gas inlet at the measurement chamber.

The time interval used for individual measurements of sensor response was $30 \mathrm{~s}$. 


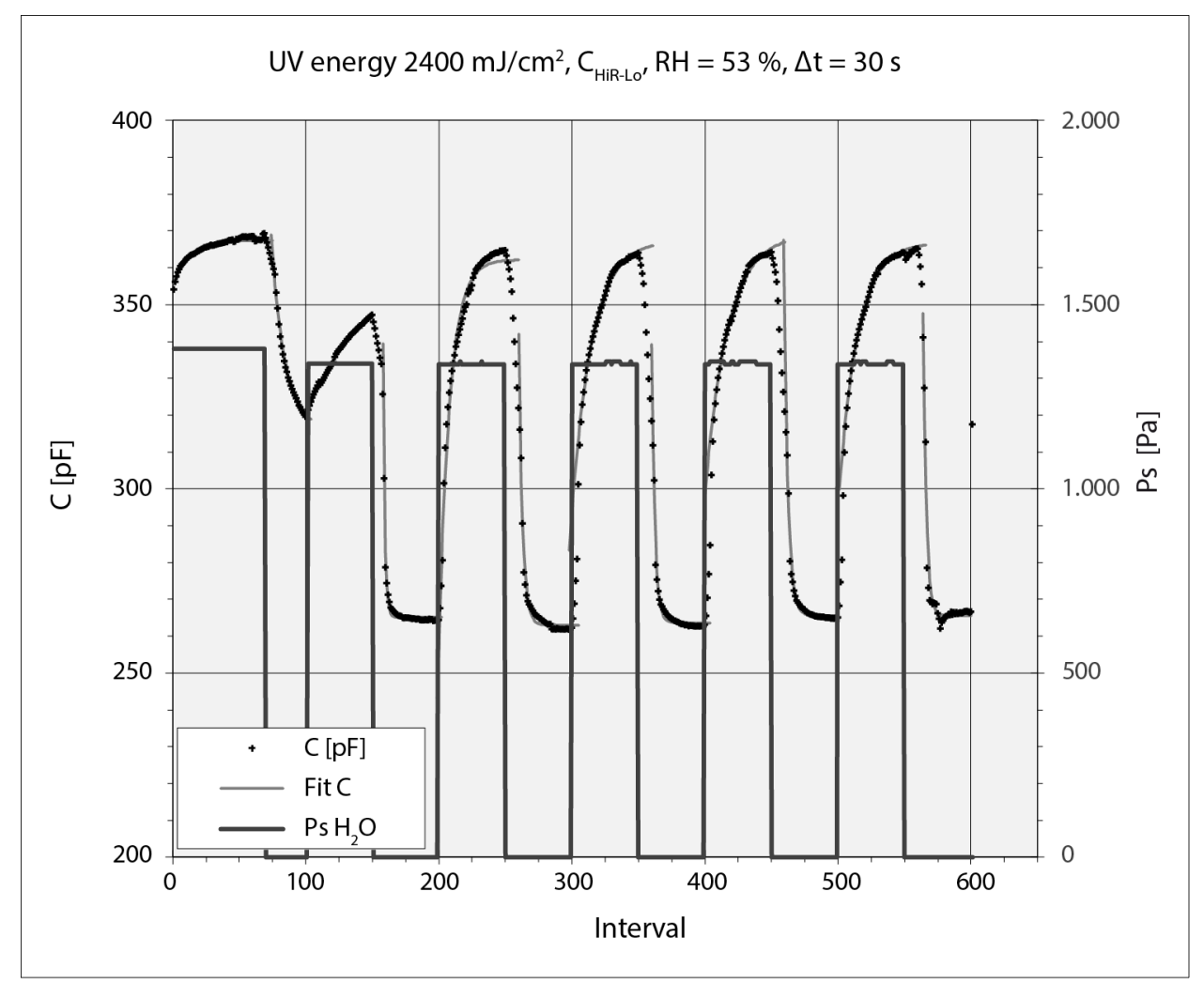

Figure 8: Sensor response to the change in relative humidity of $53 \%$ in the measurement chamber.

\section{DISCUSSION}

Differences in capacitance of sensors were due to different placements of electrodes and therefore changes in geometry and surface of electrodes. Based on very low differences between capacitance measurements of samples with dielectric layer cured at different UV energy dose we can conclude that the layer was sufficiently polymerized at UV energy of $600 \mathrm{~mJ} / \mathrm{cm}^{2}$. This figure closely reflects the recommendations of printing ink manufacturer which state that UV energy of $650 \mathrm{~mJ} / \mathrm{cm}^{2}$ is needed for complete curing of the ink. On the other hand the dissipation factor measurements showed that there was still room for improvement in terms of quality of capacitor as the samples cured at higher UV energy were closer to the value of DF=0.013 (@1 MHz) stated by the ink manufacturer.

Capacitance of sensor changes with the increase of dielectric constant of the dielectric used. The sensor response to change of relative humidity in the measurement chamber was expected as the dielectric constant of water is $\varepsilon \sim 55\left(@ 100{ }^{\circ} \mathrm{C}\right.$ ) and the dielectric ink used was specified by the manufacturer at $\varepsilon=4.76$ (@1 MHz). When using relative humidity of $53 \%$ to measure the sensor response, the base value of capacitance when the measuring chamber was purged with nitrogen gas was approximately $10 \mathrm{pF}$ greater, in comparison to measurements made at $45 \%$ relative humidity. We can conclude that the short time interval between measurements caused a temporary saturation of dielectric layer with water molecules, when the relative humidity in the measurement chamber was higher. Longer time interval between measurements could help prevent this. The results also show that sensor response was greater when higher values of relative humidity were introduced and indicate that similar results could be obtained if higher values of relative humidity were used. Sensor response to water vapour was slower when compared to response to the purge of measurement chamber with nitrogen gas. The designed sensor was reversible and did not get overly saturated when exposed to molecules of water vapour. The sensor response was more or less uniform throughout the measurements, although errors were recorded due to a malfunction of the nitrogen gas inlet 


\section{CONCLUSIONS}

Screen printed multi-layered interdigitated capacitors have been fabricated on ITO coated PE substrate with printing resolution of 300 microns using commercially available printing inks. The influence of UV energy used for dielectric layer curing and sensor geometry was considered for practical use of prepared chemical sensors. The potential of the devices for sensing has been evaluated for relative humidity capacitive sensing, by preparing samples with dielectric layers cured at different UV energy, whose electrical permittivity and thickness is sensitive to changes in relative humidity. The characterization of the sensors in transient state offered reproducible measurements when exposed to $45 \%$ and $53 \%$ relative humidity. The signal obtained from measurements was both stable and reproducible. In future efforts the described technique could be used with different dielectric materials used to study sensor response to different gas analytes, and eventually implemented in applications such as an electronic nose.

\section{ACKNOWLEDGMENTS}

The author acknowledges the support of Marta Klanjšek Gunde, National Institute of Chemistry, Marijan Maček, University of Ljubljana, Faculty of Electrical Engineering, and Matej Pivar, University of Ljubljana, Faculty of Natural Sciences and Engineering.

\section{REFERENCES}

[1] Abdul Rahman, M.S., Mukhopadhyay, S.C., Yu, P.-L.: “Novel sensors for food inspection: Modelling, Fabrication and Experimentation", (Springer, New Delhi, 2014), chapter 2.

[2] Botisalas, A., Oikonomou, P., Goustouridis, D., Ganetsos, Th., Rapis, I., Sanopoluou, M.: “A miniaturized chemocapacitor system for the detection of volatile organic compounds", Sensors and actuators B: Chemicals 177, 776-784, 2013. doi: 10.1016/j.snb.2012.11.050.

[3] Breurer, H., Breurer, R.: “Atlas klasične in modern fizike”, (DZS, 1993, Ljubljana), pages 150-157.

[4] Guadarrama-Santana, A., García-Valenzuela, A., Pérez-Jiménez, F., Polo-Parada, L.: "Interdigitated capacitance sensors in the $\mathrm{mm}$ scale with sub-femtoFarad resolution suitable for monitoring processes in liquid films", Revista Mexicana de Física 60, 451-459, 2014.

[5] Horvat, M.: "Tisk in analiza pasivnih elektronskih elementov", PhD thesis, NTF Naravoslovnotehniška fakulteta, Ljubljana, 2015.

[6] Mamishev, A.V., Sundara-Rajan, K., Yang, F., Du, Y., Zahn, M.: "Interdigital sensors and transducers", Proceedings of the IEEE International Conference on Communications 2004, (IEEE, San Diego, 2004), pages 808-844. doi:10.1109/JPROC.2004.826603.

[7] Oikonomou, P., Salapatas, A., Manoli, K., Misiakos, K., Goustouridis, D., Valamontes, E., Sanopoulou, M., Raptis, I.,Patsis, G. P.: "Chemocapacitance response simulation through polymer swelling and capacitor modelling”, Procedia Engineering 25, 423-426, 2011. doi:10.1016/j.proeng.2011.12.105.

[8] Reddy, A.S.G., Narakathu, B.B., Atashabar, M.Z., Rebros, M., Rebrosova, E., Joyce, M.K.: "Fully printed flexible humidity sensor", Procedia Engineering 25, 120-123, 2011. doi: 10.1016/j.proeng.2011.12.030.

[9] Suganuma, K.: "Introduction to printed electronics", (Springer Science+Business Media, New York, 2014), pages 1-31.

[10] Žveglič, M., Hauptman, N., Maček, M., Klanjšek Gunde, M.: "Screen-printed electrically conductive functionalities in paper substrates", Materials and technology 45(6), 627-632, 2011.

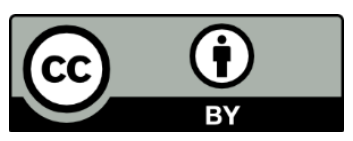

(C) 2018 Authors. Published by the University of Novi Sad, Faculty of Technical Sciences, Department of Graphic Engineering and Design. This article is an open access article distributed under the terms and conditions of the Creative Commons Attribution license 3.0 Serbia

(http://creativecommons.org/licenses/by/3.0/rs/). 